\title{
Oxygen Transfer Efficiency Evaluation of Underwater Bubbles
}

\author{
Yong-Du Jun ${ }^{1}$ and Jong-Soo Lee ${ }^{2}$ \\ ${ }^{1}$ Professor, Division of Mechanical and Automotive Engineering, Kongju National \\ University, Chungnam, Korea \\ ${ }^{2}$ Adjunct Professor, Division of Mechanical and Automotive Eningeering, Kongju \\ National University, Chungnam, Korea \\ lyjun@kogju.ac.kr, ${ }^{2}$ jonglee@kongju.ac.kr
}

\begin{abstract}
This paper presents the study results on the dissolved oxygen concentration behavior in water body with underwater bubbling. The rage of algae in dams and reservoirs around the country, especially during the summer season, is threatening the habitat environment of these water resources by significantly lowering the dissolved oxygen concentration level. Different aeration devices based on underwater bubbling are widely applied in order to remedy this problem, however, fundamental study results on the performance of these devices in terms of dissolved oxygen level improvement are rarely reported in literature. In the present study, we focus on the quantitative evaluation of oxygen transfer performance of bubble diffusion process, which is the underlying physical model of aeration devices. For this purpose, related physical laws such as Henry's law of solubility, Fick's law of diffusion along with water quality standards are reviewed and a new test apparatus is established for this study, with which both the degassing and aeration can be systematically simulated. The test apparatus is composed of a closed water tank (stainless steel; $0.65 \mathrm{~m} \times 0.65 \mathrm{~m} \times 1.0 \mathrm{~m}$ ) with depressurization and degassing feature, underwater bubbler and measurement system for temperature, pressure and oxygen level. This system is capable of maintaining minimum pressure level of 0.1 atm and the dissolved oxygen concentration level down to $2 \mathrm{mg} / \mathrm{L}$. According to the test results for a commercially available bubbler at three different air flow rates (10, 20, and 30 slpm) and at two bubbling depths of 0.4m and 0.8m, the oxygen transfer efficiency of 1.3-2.5\% are confirmed and the best performing conditions could be successfully identified.
\end{abstract}

Keywords: Dissolved oxygen, Bubble diffusion, Oxygen concentration, Oxygen transfer efficiency, Solubility

\section{Background}

Algae blooms refer to a massive range of algae which are basically plant-like planktons that photosynthesize in underwater conditions. The rage of algae in dams and reservoirs around the country, especially during the summer season, is repeatedly threatening the habitat environment of these water resources by significantly lowering the dissolved oxygen concentration level [1]. In order to remedy this problem, it is necessary to seek a countermeasure to recover the already lowered dissolved oxygen level in an effective way. Bubblers or aeration devices, frequently applied to improve the dissolved oxygen level, are the devices that supply air bubbles underwater, through which they utilize the diffusion phenomena of oxygen from inside the air

Article history:

Received (January 22, 2020), Review Result (March 1, 2020), Accepted (April 16, 2020) 
bubble to the outer water body with lowered oxygen content [2]. However, the oxygen transfer performance of these devices are not reported in a clear manner yet in the literature. Considering the use of the DC solar air pumps for bubblers as one of the cost-effective countermeasures compared to the conventional larger devices, the efficiency of the oxygen transfer performance of these devices depending on the installed conditions is of major concern.

In the present study, the behavior of dissolved oxygen concentration due to air bubbling is experimentally studied using a specially designed experimental set-up, and the performance of a tested bubble system is quantitatively evaluated based on the newly defined oxygen transfer efficiency based on the prescribed oxygen concentration range of concern.

\section{Related physical laws}

\subsection{Fick's law - The principle of mass diffusion}

Fick's $1^{\text {st }}$ law of diffusion states that the mass transfer rate is proportional to the contact area and the concentration gradient, in which the proportionality constant is referred to as the diffusion coefficient. This law applies when there exists a concentration gradient in a system. McGinnis et al. [3] proposed a modified version of the relationship that can be used for multiphase system, in which, instead of using the concentration gradient, they employed the difference in concentration between the phase, that is, between the inside the air bubble and the outside liquid water body. They also provided the corresponding proportionality constants in their work. In dealing with bubble diffusion, it is necessary to consider the bubble size and its distribution along with the shape. Assuming uniform-sized spherical bubbles are generated through a bubbler, the relation among the bubble size in terms of its diameter, surface area and the number of bubbles when a constant volume of air is supplied, can be established as shown in [Table 1], which tells that the surface area is inversely proportional to its diameter.

Table 1. Relations between the radius and the surface area of spherical bubbles given a constant volume

\begin{tabular}{|c|c|c|c|c|}
\hline Radius $(\mathrm{mm})$ & No. of bubbles & Volume $\left(\mathrm{mm}^{3}\right)$ & Surface area $\left(\mathrm{mm}^{2}\right)$ & Surface area ratio \\
\hline 10 & $1 / 1,000$ & 4.189 & 1.2566 & $1 / 10$ \\
\hline 1 & 1 & 4.189 & 12.566 & 1 \\
\hline 0.1 & 1,000 & 4.189 & 125.66 & 100 \\
\hline 0.01 & $1,000,000$ & 4.189 & $1,256.6$ & 100 \\
\hline
\end{tabular}

The residence time of the air bubble in water depends on its diameter and the depth of origin. Under the assumption that the diameter of the spherical bubble remains almost constant during the rising, it could be expected that as the bubble origin becomes deeper and deeper, the residence time would increase close to linearly, which eventually results in higher mass transfer. [Figure 1]. illustrates the bubble rise model and the free-body-diagram of underwater bubble. 


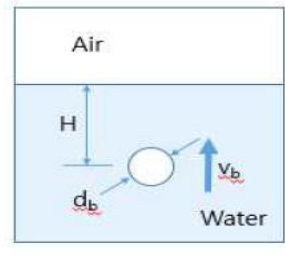

(a)

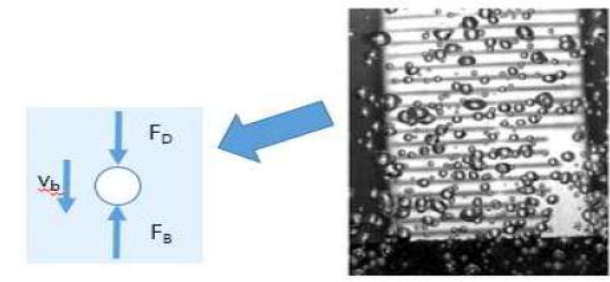

(b)

Figure 1. Bubble rise model (a) and the free-body-diagram of underwater bubble (b)

\subsection{Solubility and Henry's law}

Solubility is the property of a solute to dissolve in a solvent. It is defined as the maximum amount (gram or mol) of solute (oxygen) in a given volume of solvent (water) at a given temperature under an equilibrium condition [4]. In case of the air-water system, Henry's law for the oxygen (solute) with respect to water can be stated that the solubility of the oxygen is proportional to the partial pressure of oxygen in the air and the proportionality constant is referred to as Henry's constant. For Henry's law provides information on the possible limit of oxygen concentration in water when the partial pressure of oxygen in the air is known or given from the measurement.

\subsection{Relation between the partial pressure and the mole fraction}

For the mole fraction of species $i$ in an ideal gas mixture under a phase equilibrium is related to its pressure ratio of partial pressure of species $i$ with respect to the mixture pressure [5], the mole fraction of the oxygen in the air can be expressed in terms of the pressure ratio of the oxygen partial pressure with respect to the air pressure.

\subsection{Dissolved Oxygen (DO) contents according to water quality standard}

Fick's law states that the mass diffusion rate relies on the concentration gradient while the oxygen concentration of the air remains almost constant, it could be inferred that the mass diffusion rate should be dependent on the DO level in water. In order to come up with fair evaluation of oxygen transfer performance it is necessary to define a range of DO concentration of concern. According to the water quality standard of the Korean government [Table 2], the DO concentrations of 2,5 , and $7.5 \mathrm{mg} / \mathrm{L}$ are referred. Among these referred values, we chose the concentration range between 2.0 and $7.5 \mathrm{mg} / \mathrm{L}$ for the evaluation of the oxygen transfer performance for fair comparison.

Table 2. Dissolved Oxygen (DO) contents according to water quality standards (partially quoted from the environmental standard of water quality, ME, Korea) [6]

\begin{tabular}{|c|c|}
\hline Grade & DO(mg/L) \\
\hline Very good(Ia) & $\geq 7.5$ \\
\hline Good(Ib) & $\geq 5.0$ \\
\hline Somewhat good(II) & $\geq 5.0$ \\
\hline Normal(III) & $\geq 2.0$ \\
\hline Somewhat bad(IV) & $\geq 2.0$ \\
\hline Bad(V) & $\geq 2.0$ \\
\hline Very bad(VI) & $<2.0$ \\
\hline
\end{tabular}




\section{Methods and experiment}

An experimental apparatus to monitor the dissolved oxygen level during the bubbling process is devised, for which the degassing of dissolved gases including oxygen down to the desired level needs to be attained prior. In the present study, the degassing could be obtained through the air vent combined with underwater microbubble generation [7]. [Figure 2] illustrates the schematic diagram of the experimental setup for the present study, which is composed of a closed water tank (stainless steel; $0.65 \mathrm{~m} \times 0.65 \mathrm{~m} \times 1.0 \mathrm{~m}$ ) with depressurization and degassing feature, underwater bubbler and measurement system for temperature, pressure and oxygen level. This system is capable of maintaining minimum pressure level of $0.1 \mathrm{~atm}$ and the dissolved oxygen concentration level down to $2 \mathrm{mg} / \mathrm{L}$.

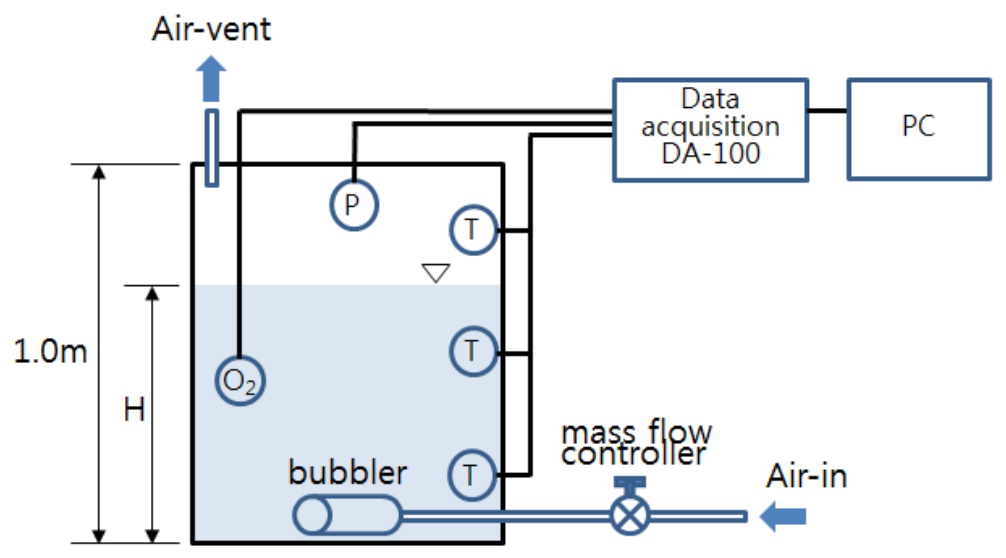

Figure 2. Diagram of the test apparatus for DO measurements

Degassing and bubbling tests are conducted for predesigned test conditions, which comprises of the air supply rates of 10,20, and $30 \mathrm{slpm}$ at two bubbling depths of $0.4 \mathrm{~m}$ and $0.8 \mathrm{~m}$, respectively. A commercial bubbler is selected for the present experiments. [Figure 3] shows a typical behavior of the measured DO concentration during the bubbling process. As mentioned ahead, the behavior of the DO concentration with respect to time or equivalently to the supplied volume of air is not linear, which suggests that we need a concentration range to be specified to give a meaningful performance comparison.

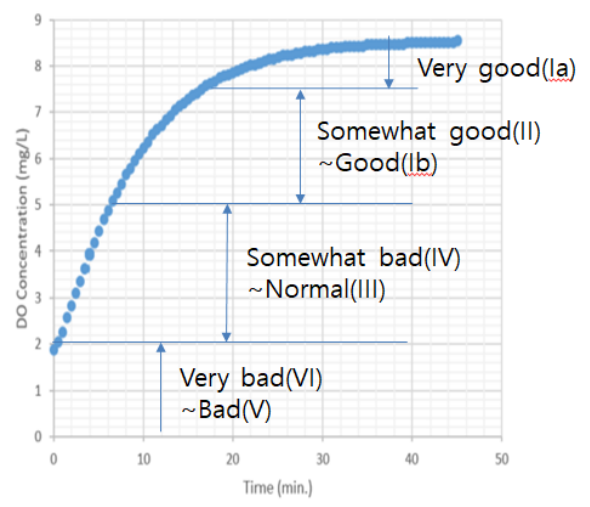

Figure 3. Typical DO measurement result during the bubbling process with corresponding water grades (V_water=338 L, Q_air $=10 \mathrm{slpm}, \mathrm{H}=0.5 \mathrm{~m}, \mathrm{~T}=25^{\circ} \mathrm{C}$ ) 
In order to evaluate the oxygen transfer performance in a quantitative manner, we propose to use a new definition of oxygen transfer efficiency with specific DO concentration range, that is, oxygen transfer efficiency, $\eta_{a \text { (conc.range) }}$, is defined as the fraction or percentile of the oxygen transferred from the air bubbles to water body with respect to the total oxygen of supplied air through aeration. In the present study, the oxygen concentration range is selected to be $2.0-7.5 \mathrm{mg} / \mathrm{L}$ which represents the grade change from IV to Ia.

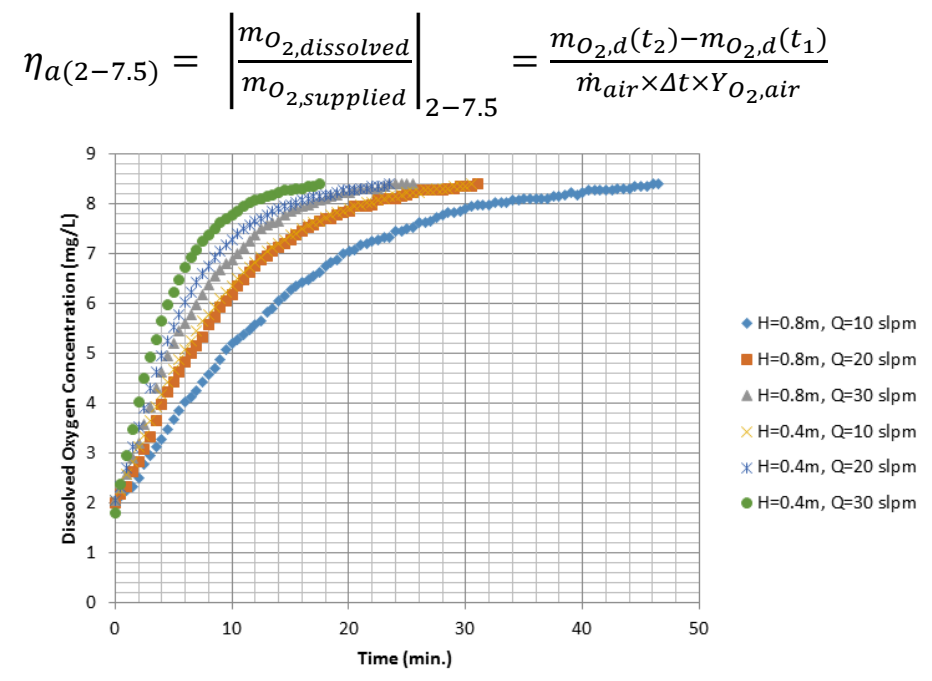

Figure 4. Measured DO with respect to time $(\mathrm{H}=0.4 \mathrm{~m}, 0.8 \mathrm{~m} ; \mathrm{Q}=10,20,30 \mathrm{slpm})$

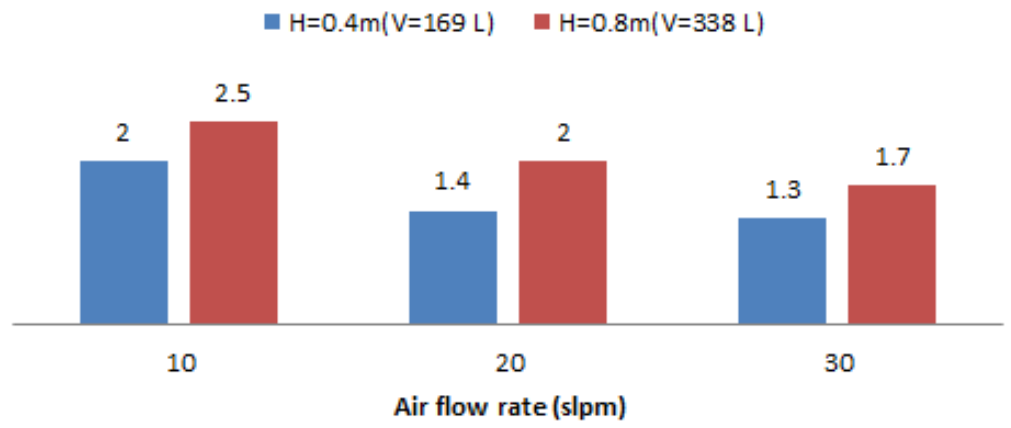

Figure 5. Oxygen transfer efficiency in the DO range from 2.0 to $7.5 \mathrm{mg} / \mathrm{L}$

\section{Results and discussions}

[Figure 4] shows the DO measurement results from the present study. From the figure, it could be said that as the supplied air mass flow rates increase, the faster the DO level saturates and, at the same token, as the bubbling depth becomes deeper the faster the DO level saturates. However, with this information only, it is not easy to say what is the best working condition and what is the efficiency of the process. [Figure 5] shows the analysis results utilizing the oxygen transfer efficiency as defined in the Eq. (1), from which it could be clearly stated that for the present test conditions herein the oxygen transfer efficiency through bubbling range from $1.3 \%$ to $2.5 \%$. It is also could be noted that both the deeper bubbling depth and the more air mass flow rates may reach the saturation conditions early, the efficiency-wise it is not always 
improving. By introducing the oxygen transfer efficiency, it was also possible to identify the working condition that may provide the highest efficiency.

\section{Conclusions}

A quantitative evaluation method of oxygen transfer performance of aeration devices including bubblers is proposed and a new test method and test apparatus featured by degassingaeration cycling is introduced and successfully demonstrated its validity through demonstration tests.

Through the present experimental study, the oxygen transfer efficiency of $1.3-2.5 \%$ between the DO level of 2.0-7.5 $\mathrm{mg} / \mathrm{L}$, depending upon the supplied air mass flow rates of 10, 20, and $30 \mathrm{slpm}$ at two bubbling depth of $0.4 \mathrm{~m}$ and $0.8 \mathrm{~m}$, is presented.

With the present approach, the conditions for highest efficiency could successfully be identified, which may be used for guidelines for selecting better design and implementation conditions for bubblers and aeration devices.

\section{Acknowledgements}

This work is supported by 2019 Kongju National University Academic Research Funding Program.

\section{References}

[1] B. Cheng, R. Xia, Y. Zhang, Z. Yang, S. Hu, F. Guo, and S. Ma, "Characterization and causes analysis for algae blooms in large river system," Sustainable Cities and Society, vol.51, 101707, (2019)

[2] J. Navisa, T. Sravya, and M. Venkatesan, "Effect of bubble size on aeration process," Asian Journal of Scientific Research, vol.7, pp.482-487, (2014)

[3] D. F. McGinnis and J. C. Little, "Predicting diffused-bubble oxygen transfer rate using the discrete-bubble model," Water Research, vol.36, pp.4627-4635, (2002)

[4] Wikipedia, the free encyclopedia, https://en.wikipedia.org/wiki/Solubility, (2020)

[5] Y. A. Cengel, M. A. Boles, and M. Kanoglu, "Thermodynamics-An Engineering Approach,” McGrawHill (2019)

[6] Environmental Standard of Water Quality, ME, Korea, http://www.me.go.kr/home/web/policy_data/read.do? menuId=10263\&seq=2, (2020)

[7] Y. D. Jun et al., "Dissolved gas discharging apparatus and dissolved gas discharging method using the same," Korean Patent 10-2020-0002833 (pending), (2020)

\section{Authors}

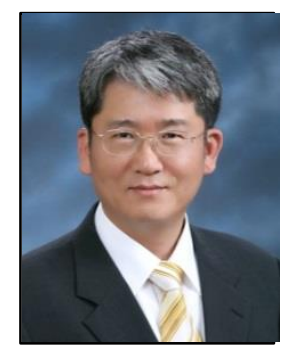

\section{Yong-Du Jun}

Professor, Division of Mechanical and Automotive Engineering, Kongju National University

$\mathrm{Ph}$. D. and MS Aerospace Engineering, University of Cincinnati, USA

BS Aeromechanical Engineering, Hankook Aviation University, Korea

Research Interest: general mechanical engineering applications for the future with an emphasis in thermal fluids. 


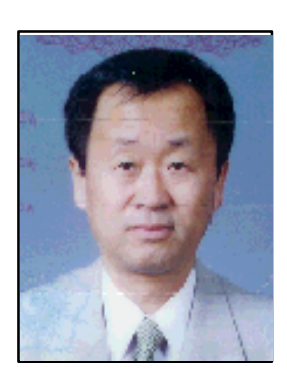

Jong-Soo Lee

Adjunct professor, Division of Mechanical and Automotive Engineering, Kongju National University

Ph. D. Applied Mechanics, University of Michigan at Ann Arbor, USA

MS \& BS Mechanical Engineering, Seoul National University, Korea

Research Interest: Quantum engineering and its applications 
Oxygen Transfer Efficiency Evaluation of Underwater Bubbles

This page is empty by intention. 